\title{
On the Dynamic Stability of a Reinforced Concrete Plate, Taking into Account the Material Creep
}

\author{
Elena Kosheleva ${ }^{1, *}$ \\ ${ }^{1}$ Moscow State University of Civil Engineering, Yaroslavskoe shosse, 26, Moscow, 129337, Russia
}

\begin{abstract}
The problem of the dynamic stability of a reinforced concrete plate armoured in two directions parallel to its edges is considered. To describe the viscoelastic properties of concrete, an integral dependence was adopted with an exponential kernel. The use of this dependence led to a linear differential equation of plate vibration. In addition to the creep of concrete, the work of the reinforcement was taken into account. The solution of the differential equation of vibrations of a plate in the form of a series with separated variables is considered, which satisfies the plate fastening conditions. Differential equations are obtained for the time function by the Bubnov-Galerkin method. The task was to find the main areas of dynamic instability. For this, the critical frequency equation was obtained. The influence of the coefficients entering into the equation of critical frequencies on the position of the main regions of dynamic instability is investigated.
\end{abstract}

\section{Problem setting}

Consider a rectangular plate loaded in its plane by forces uniformly distributed along the edges:

$$
N_{x}=N_{x 0}+N_{x t} \cos \theta t, N_{x}=N_{x 0}+N_{x t} \cos \theta t
$$

In [1], the problem of the dynamic stability of a plate whose viscoelastic properties was described by a linear differential dependence was considered. This dependence is suitable for describing the creep of old age concrete. Consider a reinforced concrete plate armoured with a different amount of reinforcement in two directions parallel to the edges of the plate. Assuming that the stresses do not exceed 0.5 at compression from destructive and 0.9 at tension, for concrete the relationship between stresses and strains, if we consider the hypothesis of A.P. Rzhanitsyn [2] on the constancy of the coefficient of shortening $v$, will take the form:

\footnotetext{
Corresponding author: elleok@mail.ru
} 


$$
\begin{gathered}
\sigma_{x}=\frac{E}{1-v^{2}}\left(\varepsilon_{x}+v \varepsilon_{y}\right)-\frac{E}{1-v^{2}} \int_{0}^{t} R(t-\theta)\left(\varepsilon_{x}+v \varepsilon_{y}\right) d \theta \\
\sigma_{y}=\frac{E}{1-v^{2}}\left(\varepsilon_{y}+v \varepsilon_{x}\right)-\frac{E}{1-v^{2}} \int_{0}^{t} R(t-\theta)\left(\varepsilon_{y}+v \varepsilon_{x}\right) d \theta \\
\tau_{x y}=\frac{E}{2(1+v)} \gamma_{x y}-\frac{E}{2(1+v)} \int_{0}^{t} R(t-\tau) \gamma_{x y} d \theta
\end{gathered}
$$

In these relations, the kernel is taken in the form of an exponential function:

$$
R(t-\theta)=\frac{E-H}{n E} e^{-\frac{t-\theta}{n}}
$$

where $\mathrm{n}$-the relaxation time, $\mathrm{E}$-the instantaneous modulus of elasticity, and $\mathrm{H}$ - the longterm modulus of elasticity. For the stresses of the reinforced steel we take the relations [3]:

$$
\sigma_{x}^{a}=\frac{E_{a}}{1-v_{a}^{2}}\left(\varepsilon_{x}+v \varepsilon_{y}\right), \sigma_{y}^{a}=\frac{E_{a}}{1-v_{a}^{2}}\left(\varepsilon_{y}+v \varepsilon_{x}\right) .
$$

In the bending equation of the plate [3], adding the forces of inertia and forces (1), we obtain the equation of plate vibrations:

$$
\begin{aligned}
& \left(B E+B_{a}^{x} E_{a}\right) \frac{\partial^{4} w}{\partial x^{4}}+\left[E_{a} v_{a}\left(B_{a}^{x}+B_{a}^{y}\right)+2 B E\right] \frac{\partial^{4} w}{\partial x^{2} \partial y^{2}}+\left(B E+B_{a}^{y} E_{a}\right) \frac{\partial^{4} w}{\partial y^{y}}- \\
& E B\left[\int_{0}^{t} R(t-\theta) \frac{\partial^{4} w}{\partial x^{4}} d \theta+2 \int_{0}^{t} R(t-\theta) \frac{\partial w^{4}}{\partial x^{2} \partial y^{2}} d \theta+\int_{0}^{t} R(t-\theta) \frac{\partial^{4} w}{\partial y^{4}} d \theta\right]+ \\
& \left(N_{x 0}+N_{x t} \cos \theta t\right) \frac{\partial^{2} w}{\partial x^{2}}+\left(N_{y 0}+N_{y t} \cos \theta t\right) \frac{\partial^{2} w}{\partial y^{2}}+m \frac{\partial^{2} w}{\partial t^{2}}=0,
\end{aligned}
$$

where $\quad B_{a}^{x}=\frac{I_{a}^{x}}{1-v_{a}^{2}}, B=\frac{I_{a}^{x}}{1-v_{a}}, B=\frac{h^{3}}{1-v^{2}}, I_{a}^{x}, I_{a}^{y} \quad-\quad$ moments of inertia of the cross sections of the reinforcement steel per unit length, $h$ is the thickness of the plate.

\section{Solution technique}

The solution to this equation can be found by separating the variables:

$$
w=\sum f_{i}(t) X_{i}(x) Y_{i}(y)
$$

where $f_{i}(t)$ - the unknown time functions, $X_{i}(x)$ and $Y_{i}(y)$ - functions fulfilling the boundary conditions along the edges of the rectangular outline relative to $w$. By inserting the solution (5) to (4) and by utilizing the Bubnov-Galerkin method, an equation with alternating coefficients is obtained:

$$
f^{\prime \prime \prime}+\alpha f^{\prime \prime}+\varphi(\gamma-2 \mu \cos \tau) f^{\prime}+\alpha \varphi\left(\chi-2 \mu \cos \tau+\frac{2}{\alpha} \mu \sin \tau\right) f=0
$$


where $\tau=\theta t$ is a dimensionless time,

$$
\begin{gathered}
\Omega^{2}=\omega^{2}\left(1-\frac{N_{x 0} N_{2 *}+N_{y 0} N_{1 *}}{N_{1 *} N_{2 *}}\right), \mu=\frac{1}{2} \frac{N_{x t} N_{2 *}+N_{y t} N_{1 *}}{N_{1 *} N_{2 *}-N_{x 0} N_{2 *}-N_{y 0} N_{1 *}}, \eta=\frac{D_{a} I_{5}}{D I_{1}}, \\
\gamma=\frac{N_{1 *} N_{2 *}(1+\eta)-N_{x 0} N_{2 *}-N_{y 0} N_{1 *}}{N_{1 *} N_{2 *}-N_{x 0} N_{2 *}-N_{y 0} N_{1 *}}, \quad \chi=\frac{N_{1 *} N_{2 *}\left(\xi_{1}+\eta\right)-N_{x 0} N_{2 *}-N_{y 0} N_{1 *}}{N_{1 *} N_{2 *}-N_{x 0} N_{2 *}-N_{y 0} N_{1 *}},
\end{gathered}
$$

$\Omega$ is the frequency of the natural vibrations of the plate loaded by the forces $N_{x 0}$ and $N_{y 0}$, $N_{I^{*}}$ and $N_{2^{*}}$ - the critical values of the forces $N_{x 0}$ and $N_{y 0}$ for their independent static action, $\mu$ is the excitation coefficient, $\chi$ is the dimensionless parameter, the magnitude of which is affected by $\eta$, from the characteristics of the reinforcement steel,

$$
\begin{gathered}
N_{1 *}=\frac{I_{1}}{I_{3}} D, N_{2 *}=\frac{I_{1}}{I_{4}} D, \alpha=\frac{1}{n \theta}, \varphi=\frac{\Omega^{2}}{\theta^{2}}, \delta=\frac{B_{a}^{y}}{B_{a}^{x}}, B H=D \xi_{1}, \xi_{1}=\frac{H}{E}, \\
B E=D, \\
I_{1}=\int_{0}^{a} \int_{0}^{b}\left(\frac{d^{4} X}{d x^{4}} Y+2 \frac{d^{2} X}{d x^{2}} \frac{d Y^{2}}{d y^{2}}+\frac{d Y^{4}}{d y^{4}} X\right) X Y d x d y, I_{2}=\int_{0}^{a} \int_{0}^{b} X^{2} Y^{2} d x d y, \\
I_{3}=-\int_{0}^{a} \int_{0}^{b} X \frac{d^{2} X}{d x^{2}} Y^{2} d x d y, I_{4}=-\int_{0}^{a} \int_{0}^{b} X^{2} \frac{d^{2} Y}{d y^{2}} Y d x d y, \quad \int_{0}^{a} \int_{0}^{b}\left(\frac{d^{4} X}{d x^{4}} Y+v_{a}(1+\delta) 2 \frac{d^{2} X}{d x^{2}} \frac{d Y^{2}}{d y^{2}}+\delta \frac{d Y^{4}}{d y^{4}} X\right) X Y d x d y, \quad
\end{gathered}
$$

$\omega$ frequency of natural oscillations of an unloaded plate. The coefficients of equation (7) affect its solutions, which can increase indefinitely and occupy regions on the plane $\mu, \frac{\theta}{2 \Omega}$. The main instability regions can be found [4], looking for the solution of equation (7) in the form of a series with a period $T$ :

$$
f(t)=\sum_{k=1,3,5}^{\infty}\left(a_{k} \sin \frac{k \tau}{2}+b_{k} \cos \frac{k \tau}{2}\right) .
$$

Substituting (10) into equation (7) and equating the coefficients for the same $\sin \frac{k \tau}{2}$ and $\cos \frac{k \tau}{2}$, we obtain a system of linear equations for $a_{k}$ and $b_{k}$. Having formed the determinant from the coefficients of the system and equating it to zero, we obtain the equation of critical frequencies: 


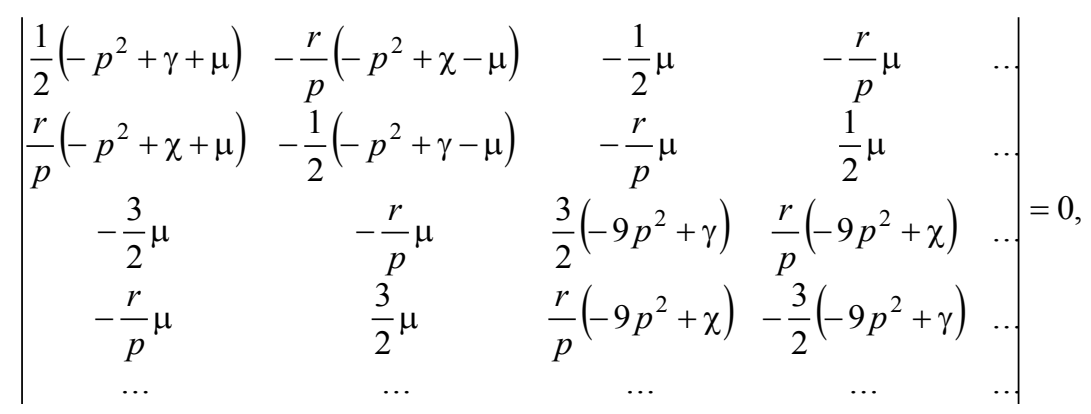

where $p=\frac{\theta}{2 \Omega}, \varphi=\frac{1}{4 p^{2}}, \quad r=\frac{1}{2 n \Omega}$.

\section{The findings}

Let us investigate the influence of the coefficients on the position of the main instability region. To determine its position, we solve equation (9). This equation includes the coefficient $r$, depending on $n=\frac{\psi}{2 \pi \omega} \frac{H}{E}$, where $\psi$ is the absorption coefficient, which is equal to 0.2-0.6 for reinforced concrete; the oscillation frequency $\omega, \frac{E}{H}$ is assumed to be 1.1-1.2. Substituting $n$ in $r$, we obtain $r=\frac{\pi E}{\psi H}$.. With this change $\psi$, value $r$ will decrease from $15.71 \frac{E}{H}$ to $5.24 \frac{E}{H}$. Proceeding from the results of [1], it can be noted that such a change in $r$ will lead to an increase in the minimum value of the excitation coefficient, and, consequently, to an increase in the amplitudes of the periodic load. When studying the influence of $\zeta_{1}$ and $\eta$, we take $r=5.24 \frac{E}{H}$. Let us establish the limits of variation of these quantities. The coefficient $\zeta_{1}$ varies from 0.91 to 0.833 . The value of $\eta$ depends on the ratio of the moduli of elasticity of the plate material and the reinforcement steel, the shape of the oscillation, and the percentage of reinforcement. Convert the coefficient

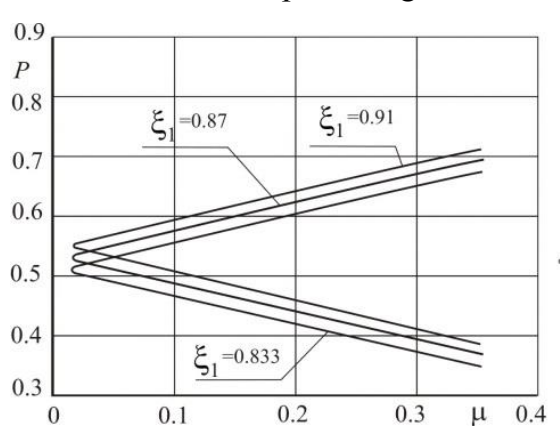

Fig. 1. The boundaries of the main instability regions $\eta=0.2$ and $\beta=0.2$$$
\text { regions of dynacin instaility, taking }
$$

regions of dynamic instability, taking $\zeta_{1}=0.833, \beta=0.2$ and $\beta=0.4$, and $\eta$, depending 
directly on the percentage of reinforcement, will be changed in steps of 0.2 . The results of the calculations are shown in Figures 2 and 3

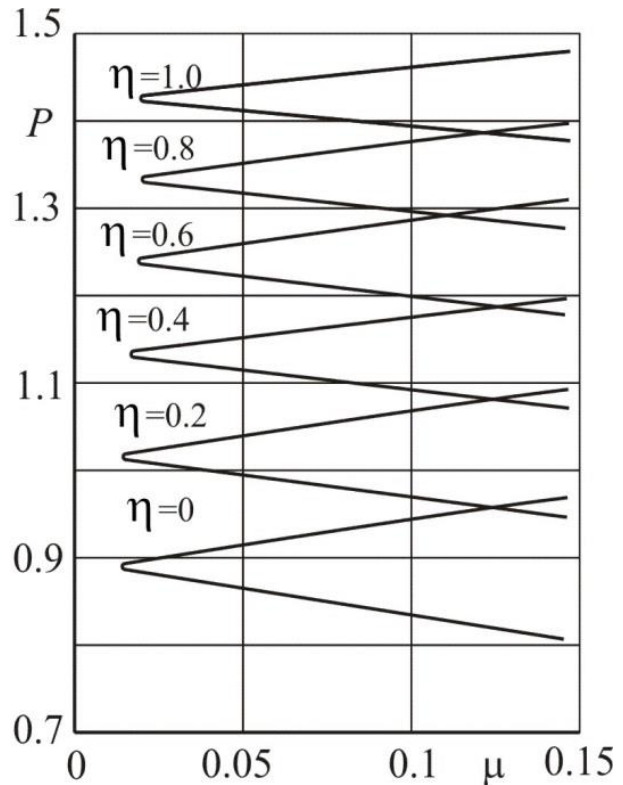

Fig. 2. The boundaries of the main instability regions $\zeta_{1}=0.833, \beta=0.2$

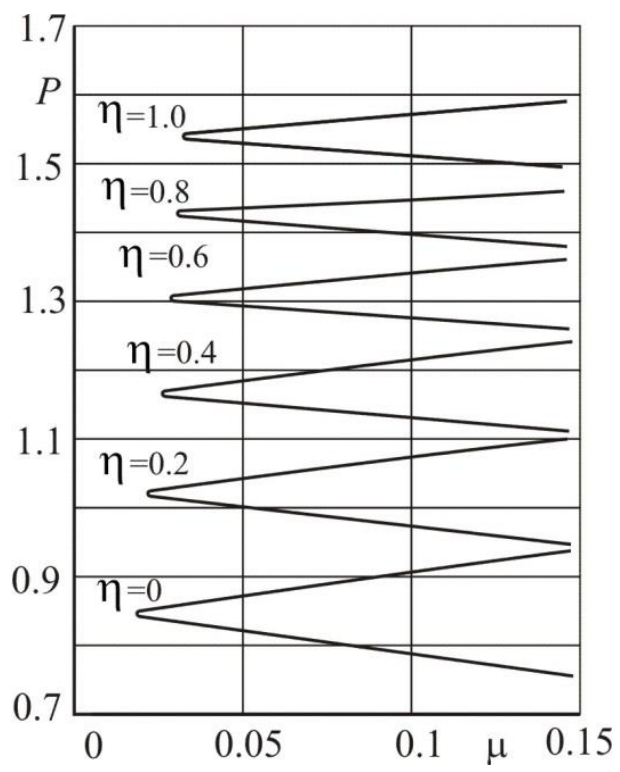

Fig. 3. The boundaries of the main instability regions $\zeta_{1}=0.833, \beta=0.4$

The depicted regions are spaced from the $p$ axis at some distance, which determines the minimum value of the excitation coefficient $\mu$. With increasing $\eta$, this distance increases, which leads to an increase in the minimum value of the excitation coefficient $\mu$, and in Figure 3 this occurs more intensively, since the constant load has increased. In addition, with increasing $\eta$, and, consequently, the percentage of reinforcement, the regions move upwards, towards increasing values $p$, which leads to an increase in the frequency of the periodic load, which can cause a loss of dynamic stability. It should be noted that as $\eta$ increases, the distance to which the graph moves upward decreases. With increasing compressive forces, the decrease in this distance occurs more intensively.

\section{Conclusion}

The effect of the parameters of the kernel of a viscoelastic material of a rectangular reinforced concrete plate and the percentage of reinforcement on the position of the main region of dynamic instability is investigated. It was revealed that with an increase in the percentage of reinforcement with an unchanging constant component of the load, an increase in the minimum value, and, hence, in the amplitudes of the variable load components, is capable of causing a loss of dynamic stability. Recently, articles have been published in which new results have been reported on the investigation of viscoelastic properties of materials and the development of methods for calculating the corresponding structures [5-8etc.] 


\section{References}

1. E.L. Kosheleva MATEC Web of Conferences 117, 00086 (2017)

2. A.R. Rzhanitsyn, Creep theory. (Stroyizdat, 1968)

3. G.A. Teters in: Creep of building materials and structures. (Stroyizdat, 1964)

4. V.V. Bolotin, Dynamic Stability of Elastic Systems (GITL, 1956)

5. N.Yu.Tsybin, R.A.Turusov, V.I.Andreev, Procedia engineering 153 ( 2016 )

6. V.I. Andreev, A.S. Chepurnenko, B.M. Yazyev,. AMR, 900 (2014)

7. V.I. Andreev, A.S. Chepurnenko, B.M. Yazyev, AMR 1004-1005 (2014)

8. V.I. Andreev, A.S. Chepurnenko, B.M. Yazyev, A.N. MATEC Web of Conferences 67,06059 (2016) 